\title{
LMI Based Principles in Strictly Metzlerian Systems Control Design
}

\author{
Dušan Krokavec $(\mathbb{D}$ and Anna Filasová \\ Department of Cybernetics and Artificial Intelligence, Faculty of Electrical Engineering and Informatics, \\ Technical University of Košice, Letná 9, 04200 Košice, Slovakia
}

Correspondence should be addressed to Dušan Krokavec; dusan.krokavec@tuke.sk

Received 5 August 2017; Revised 15 January 2018; Accepted 22 April 2018; Published 17 July 2018

Academic Editor: Marzio Pennisi

Copyright (C) 2018 Dušan Krokavec and Anna Filasová. This is an open access article distributed under the Creative Commons Attribution License, which permits unrestricted use, distribution, and reproduction in any medium, provided the original work is properly cited.

\begin{abstract}
The paper is concerned with the design requirements that relax the existing conditions reported in the previous literature for continuous-time linear positive systems, reformulating the linear programming approach by the linear matrix inequalities principle. Incorporating an associated structure of linear matrix inequalities, combined with the Lyapunov inequality guaranteeing asymptotic stability of positive system structures, the conditions are presented, with which the state-feedback controllers and the system state observers can be designed. A numerical example illustrates the proposed conditions.
\end{abstract}

\section{Introduction}

Positive systems are often found in the modeling and control of engineering and industrial processes, whose state variables represent quantities that do not have meaning unless they are nonnegative [1]. The mathematical theory of Metzler matrices has a close relationship to the theory of positive linear continuous-time dynamical systems, since in the statespace description form the system dynamics matrix of a positive systems is Metzler and the system input and output matrices are nonnegative matrices. In abbreviated terms, such continuous-time linear systems are denoted in the following as Metzlerian systems. Stability and stabilization of such systems are reported, e.g., in [2-6] and various methods based on linear programming (LP) and linear matrix inequalities (LMI) are considered for positive stabilization and observer designs, as it is further elaborated in subsequent paragraphs.

The task of the state observer design for positive linear system is solved in [7] using the coordinates transformation. If the coordinate transform matrix is a solution of the specified Silvester equation and its inverse is nonnegative, the positive observer is constructed only for transformed state coordinates. Another proposed way is to transform system into controllable and uncontrollable parts to obtain a reduced order realization, suitable for positive observer design. The second group of methods represents [8], where LMI conditions to state observer design for positive linear systems are presented, but the observer is not of Luenberger type since the standard observer gain matrix does not specify the observer dynamics and an additive matrix is used to define the observer stability.

The most similar idea to the one presented in this paper is the approach given in $[9,10]$, using formulation based on the set of LMIs. These approaches are considered for both positive and nonpositive systems. However, since nondiagonal matrix variables are exploited, they do not in general guarantee diagonally dominant Metzler structure of the closed-loop system matrix. The approach of the present paper makes it possible to achieve this additional constraint, if required.

The linear programming approach was effectively used for static output feedback design of positive systems in [11, $12]$, and $[13,14]$ consider both positive observer and control design with bounded control constraint.

The main motivation of this paper is to give design conditions for stabilization of linear positive continuous-time systems by the state-feedback, as well as the conditions for Lunberger state observers design in the same systems structures, using the formulation based on strict LMIs. Analyzing structures of the closed-loop system matrix, and the observer 
system matrix, the resulting algebraic constraints, which lead to strictly Metzler matrices, are formulated as a set of LMIs with diagonal matrix variables. This set is extended by an LMI, reflecting the Lyapunov stability condition [15]. Since the stability is posed as an LMI, constraints implying from the strictly Metzler matrix structure in the form of LMIs results also in the LMI formulation [16]. Advantages may also be added to the fact that these conditions can be adapted to the synthesis of nonnegative gain matrices of the regulator or estimator, as well as for the synthesis resulting in a Metzler matrix structure, if the conditions for its zero nondiagonal elements are satisfied. Preferring LMI structure, the proofs are of standard way in the sense of Lyapunov principle and reflect the facts when optimization in positive systems has LMIs representation.

The paper is organized as follows. After the Introduction, Section 2 presents some preliminaries, including the characterization of positive systems. A newly introduced set of LMIs, describing the design conditions for strictly Metzlerian SISO systems, is theoretically substantiated and proven in Section 3 and, subsequently, the design conditions for strictly Metzlerian MIMO systems are generalized in Section 4. An example is provided to demonstrate the proposed approach in Section 5, while Section 6 draws some conclusions.

Used notations are conventional so that $\boldsymbol{x}^{T}, \boldsymbol{X}^{T}$ denote transpose of the vector $\boldsymbol{x}$ and matrix $\boldsymbol{X}$, respectively, $\boldsymbol{y} \in$ $\mathbb{R}_{+}^{p}, \boldsymbol{Y} \in \mathbb{R}_{+}^{p \times q}$ denote nonnegative vector and nonnegative matrix, $\boldsymbol{X}=\boldsymbol{X}^{T}>0$ means that $\boldsymbol{X}$ is symmetric positive definite matrix, $\rho(*)$ indicates the eigenvalue spectrum of a square matrix, the symbol $\boldsymbol{I}_{n}$ marks the $n$-th order unit matrix, diag[.] enters up a diagonal matrix, $\mathbb{R}^{n}, \mathbb{R}^{n \times r}$ refer to the set of all $n$-dimensional real vectors and $n \times r$ real matrices, respectively, and $\mathbb{R}_{n}^{n}, \mathbb{R}_{+}^{n \times r}$ refer to the set of all $n$-dimensional real nonnegative vectors and $n \times r$ real nonnegative matrices, respectively.

\section{System Description}

In the following the Metzlerian class of positive linear dynamical systems is considered where state-space description is

$$
\begin{aligned}
& \dot{\boldsymbol{q}}(t)=\boldsymbol{A} \boldsymbol{q}(t)+\boldsymbol{B} \boldsymbol{u}(t), \\
& \boldsymbol{y}(t)=\boldsymbol{C} \boldsymbol{q}(t),
\end{aligned}
$$

where $\boldsymbol{q}(t) \in \mathbb{R}^{n}, \boldsymbol{u}(t) \in \mathbb{R}^{r}, \boldsymbol{y}(t) \in \mathbb{R}^{m}$ stand for state, control input, and measurable output, $\boldsymbol{A} \in \mathbb{R}^{n \times n}, \boldsymbol{B} \in \mathbb{R}^{n \times r}$, $C \in \mathbb{R}^{m \times n}$.

Definition 1 ([17], positive linear system). The linear system $(1),(2)$ is said to be positive if and only if for every nonnegative initial state and for every nonnegative input its state and output are nonnegative.

Definition 2. A matrix $\boldsymbol{Y} \in \mathbb{R}_{+}^{p \times q}$ and a vector $\boldsymbol{y} \in \mathbb{R}_{+}^{p}$ are nonnegative if all its entries are nonnegative and at least one is positive.

Definition 3 (see [18-20]). A square matrix $A \in \mathbb{R}^{n \times n}$ is Metzler matrix if its off-diagonal elements are nonnegative.
A Metzler matrix $A$ is called strictly Metzler if its diagonal elements are negative and its off-diagonal elements are positive. A Metzler matrix is stable if it is strictly Metzler and Hurwitz.

Proposition 4 (see [21]). A Metzler matrix $\boldsymbol{A} \in \mathbb{R}^{n \times n}$ is stable if and only if all principal minors of the matrix $-\boldsymbol{A}$ are positive.

Proposition 5 (see $[22,23])$. A square matrix $\boldsymbol{A} \in \mathbb{R}^{n \times n}$ is said to be strictly diagonally dominant if

$$
\left|a_{i i}\right|>\sum_{j \neq i}\left|a_{i j}\right| \quad \forall i \in\langle 1, n\rangle
$$

where $a_{i j}$ denotes the entry in the ith row and jth column.

If the matrix is strictly diagonally dominant and all its diagonal elements are negative, then the real parts of all its eigenvalues are negative. A strictly Metzler matrix is stable if it is strictly diagonally dominant.

To highlight some features of positive systems, presented exposition is placed within the framework introduced by the monograph [17], p. 8 and 41.

Proposition 6 (see [17]). A solution $\boldsymbol{q}(t)$ of (1) for $t \geq 0$ is asymptotically stable and positive if $\boldsymbol{A}$ is stable Metzler matrix, $\boldsymbol{B} \in \mathbb{R}_{+}^{n \times r}$ is nonnegative matrix and $\boldsymbol{q}(t) \in \mathbb{R}_{+}^{n}$ for given $\boldsymbol{u}(t) \in$ $\mathbb{R}_{+}^{r}$ and $\boldsymbol{q}(0) \in \mathbb{R}_{+}$. The linear system (1), (2) is asymptotically stable and positive if $\boldsymbol{A}$ is stable Metzler matrix, $\boldsymbol{B} \in \mathbb{R}_{+}^{n \times r}, \boldsymbol{C} \in$ $\mathbb{R}_{+}^{m \times n}$ are nonnegative matrices and $\boldsymbol{y}(t) \in \mathbb{R}_{+}^{m}$ for all $\boldsymbol{u}(t) \in$ $\mathbb{R}_{+}^{r}$ and $\boldsymbol{q}(0) \in \mathbb{R}_{+}$. The linear system (1), (2) is asymptotically stable and externally positive if $\boldsymbol{A}$ is stable Metzler matrix and $\boldsymbol{B} \in \mathbb{R}_{+}^{n \times r}, \boldsymbol{C} \in \mathbb{R}_{+}^{m \times n}$ are nonnegative matrices.

In process control, (2) defines the measurement subsystem on the plant and $\boldsymbol{y}(i)$ defines the output variables, whose values must be complied. If the $C$ matrix would be not nonnegative, some of the output variables could be negative. Thus, considering the nonnegative state variables and $C \in$ $\mathbb{R}_{+}^{m \times n}$, the output variables are also nonnegative.

Proposition 7 ([24], Lyapunov inequalities). Autonomous system (1) is asymptotically stable if there exist symmetric positive definite matrices $\boldsymbol{P}, \boldsymbol{Q} \in \mathbb{R}^{n \times n}$ or $\boldsymbol{V}, \boldsymbol{U} \in \mathbb{R}^{n \times n}$ such that

$$
\begin{aligned}
\boldsymbol{P} & =\boldsymbol{P}^{T}>0, \\
\boldsymbol{Q} & =\boldsymbol{Q}^{T}>0, \\
\boldsymbol{A P}+\boldsymbol{P} \boldsymbol{A}^{T}+\boldsymbol{Q} & \prec 0, \\
\boldsymbol{V} & =\boldsymbol{V}^{T} \succ 0, \\
\boldsymbol{U} & =\boldsymbol{U}^{T} \succ 0, \\
\boldsymbol{V A}+\boldsymbol{A}^{T} \boldsymbol{V}+\boldsymbol{U} & \prec 0 .
\end{aligned}
$$

Note that the equivalent statements (4) and (5) are given to circumvent formulations with inverse matrices in the following sections, since direct use (4) leads to bilinear matrix 
inequality in the design of the observer, and direct use (5) leads to bilinear matrix inequality in the synthesis of the regulator. It is easily verifiable that premultiplying the left side and postmultiplying the right side by the matrix $\boldsymbol{P}=\boldsymbol{V}^{-1}$ and setting $\mathbf{Q}=\boldsymbol{V}^{-1} \boldsymbol{U} \boldsymbol{V}^{-1}$; then (5) implies (4).

Lemma 8. If $\boldsymbol{X} \in \mathbb{R}^{n \times n}$ is a symmetric positive definite matrix, it yields the following relation:

$$
\begin{aligned}
\boldsymbol{X} & =\boldsymbol{X}^{T}>0 \Longleftrightarrow \\
\frac{1}{2} \boldsymbol{X}+\frac{1}{2} \boldsymbol{X}^{T} & =\frac{1}{2} \boldsymbol{X}+(*)>0,
\end{aligned}
$$

where $*$ denotes the symmetric item $(1 / 2) \boldsymbol{X}^{T}$ to $(1 / 2) \boldsymbol{X}$.

Proof. Since it yields

$$
\begin{aligned}
& \boldsymbol{X}=\boldsymbol{X}^{T}>0, \\
& \boldsymbol{X}=\frac{1}{2} \boldsymbol{X}+\frac{1}{2} \boldsymbol{X}=\frac{1}{2} \boldsymbol{X}+\frac{1}{2} \boldsymbol{X}^{T}>0
\end{aligned}
$$

(7) and (8) imply (6). This concludes the proof.

Definition 9 ([25], congruent modulo $n$ ). Let $n$ be a fixed positive integer. Two integers $i$ and $h$ are congruent modulo $n$ if they differ by an integral multiple of the integer $n$ (they leave the same remainder when divided by $n$ ). If $i$ and $h$ are congruent modulo $n$, the expression $(i=h)_{\bmod n}$ is called a congruence, and the number $n$ is called the modulus of the congruence.

Note that the statement $(i=h)_{\bmod n}$ is equivalent to the statement " $(i-h)$ is divisible by $n$ " or to the statement "there is an integer $m$ for which $i-h=m n$ " and the word "modulo" generally means "to the modulus".

Definition 10 (see [26]). Let $S=\{0,1,2, \ldots, n-1\}$ be the complete set of residues for any positive integer $n$. The addition modulo $n$ on $S$ is $(h+k)_{\bmod n}=r$, where $r$ is the element of $S$ to which the result of the usual sum of integers $h$ and $k$ is congruent modulo $n$.

Corollary 11. The problem of indexing in this paper is that the rows and columns of a square matrix of dimension $n \times n$ are generally denoted from 1 to $n$ and not from 0 to $n-1$. From this reason let $S=\{0,1,2, \ldots, n\}$ be the complete set of residues for any positive integer $n+1$. Then the addition modulo $n+1$ on $S$ is in the following defined as $(i+h)_{\bmod n+1}=r+1$, where $r$ is the element of $S$ to which the result of the usual sum of integers $h$ and $k$ is congruent modulo $n+1$. The used shorthand symbolical notation for $(1+h)_{\bmod n+1}=r+1$ is so $(i+h)_{(1 \longleftrightarrow n) / n}=r+1$.

\section{SISO Strictly Metzlerian Systems}

The systems under consideration in this section are linear single-input, single-output (SISO) continuous-time dynamic systems represented in state-space form as

$$
\begin{aligned}
& \dot{\boldsymbol{q}}(t)=\boldsymbol{A} \boldsymbol{q}(t)+\boldsymbol{b} u(t), \\
& y(t)=\boldsymbol{c}^{T} \boldsymbol{q}(t),
\end{aligned}
$$

where $\boldsymbol{q}(t) \in \mathbb{R}^{n}$ is the vector of the system state variables, $u(t) \in \mathbb{R}, y(t) \in \mathbb{R}$ are the input and output variables, $\boldsymbol{A} \in \mathbb{R}^{n \times n}$ is Metzler matrix, $\boldsymbol{b} \in \mathbb{R}_{+}^{n}, \boldsymbol{c} \in \mathbb{R}_{c}^{n}$ are nonnegative vectors, the pair $(\boldsymbol{A}, \boldsymbol{b})$ is controllable, and the pair $\left(\boldsymbol{A}, \boldsymbol{c}^{T}\right)$ is observable.

Note that if $\boldsymbol{A} \in \mathbb{R}^{n \times n}$ is Metzler matrix, and $\boldsymbol{b} \in \mathbb{R}_{+}^{n}$, $c \in \mathbb{R}_{+}^{n}$ are nonnegative vectors, (9) and (10) are Metzlerian system.

3.1. State Controller Design. Considering system (9), (10), the full state control law is defined as

$$
\boldsymbol{u}(t)=-\boldsymbol{k}^{T} \boldsymbol{q}(t),
$$

where $\boldsymbol{k} \in \mathbb{R}_{+}^{n}$ is the gain vector. Thus, using (9), (11), it yields

$$
\begin{aligned}
& \dot{\boldsymbol{q}}(t)=\left(\boldsymbol{A}-\boldsymbol{b} \boldsymbol{k}^{T}\right) \boldsymbol{q}(t)=\boldsymbol{A}_{c} \boldsymbol{q}(t), \\
& y(t)=\boldsymbol{c}^{T} \boldsymbol{q}(t),
\end{aligned}
$$

where

$$
\boldsymbol{A}_{c}=\boldsymbol{A}-\boldsymbol{b} \boldsymbol{k}^{T}
$$

is the closed-loop system dynamics matrix.

Note that the controller has to be designed not only to stabilize the system, but also to render the stable closed-loop system matrix strictly Metzlerian.

The following theorem defines the LMI conditions to obtain a strictly Metzler matrix $\boldsymbol{A}_{c}$ but does not guarantee that $\boldsymbol{A}_{c}$ is stable strictly Metzler matrix.

Theorem 12. The closed-loop system (12), (13) is strictly Metzlerian if system (9), (10) is strictly Metzlerian and there exists a positive definite diagonal matrix $K \in \mathbb{R}^{n \times n}$ such that for $h=1,2, \ldots n-1$,

$$
\begin{aligned}
& \frac{1}{2}\left(\boldsymbol{A}(i, i)_{(1 \longleftrightarrow n)}-\boldsymbol{B}_{d} \boldsymbol{K}\right)+(*) \prec 0, \\
& \frac{1}{2}\left(\boldsymbol{T}^{h} \boldsymbol{A}(i, i+h)_{(1 \longleftrightarrow n) / n} \boldsymbol{T}^{h T}-\boldsymbol{T}^{h} \boldsymbol{B}_{d} \boldsymbol{T}^{h T} \boldsymbol{K}\right)+(*) \\
& \quad>0, \\
& \boldsymbol{K}=\operatorname{diag}\left[\begin{array}{llll}
k_{1} & k_{2} & \cdots & k_{n}
\end{array}\right]>0,
\end{aligned}
$$


where

$$
\begin{aligned}
& \boldsymbol{T}=\left[\begin{array}{ccccc}
0 & 0 & \cdots & 0 & 1 \\
1 & 0 & \cdots & 0 & 0 \\
& & \ddots & & \\
0 & 0 & \cdots & 1 & 0
\end{array}\right], \\
& \boldsymbol{T}^{-1}=\boldsymbol{T}^{T}, \\
& \boldsymbol{b}^{T}=\left[\begin{array}{llll}
b_{1} & b_{2} & \cdots & b_{n}
\end{array}\right], \\
& \boldsymbol{B}_{d}=\operatorname{diag}\left[\begin{array}{llll}
b_{1} & b_{2} & \cdots & b_{n}
\end{array}\right]=\operatorname{diag}\left[\left\{b_{i}\right\}_{i=1, \ldots, n}\right], \\
& \boldsymbol{A}(i, i)_{1 \longleftrightarrow n}=\operatorname{diag}\left[\begin{array}{llll}
a_{11} & a_{22} & \cdots & a_{n n}
\end{array}\right] \\
& =\operatorname{diag}\left[\left\{a_{i i}\right\}_{i=1, \ldots, n}\right] \text {, } \\
& \boldsymbol{A}(i, i+h)_{(1 \longleftrightarrow n) / n} \\
& =\operatorname{diag}\left[\begin{array}{lllllll}
a_{1,1+h} & a_{2,2+h} & \cdots & a_{n-h, n} & a_{n-h+1,1} & \cdots & a_{n, h}
\end{array}\right],
\end{aligned}
$$

while $\boldsymbol{A}(i, i)_{1 \longleftrightarrow n}, \boldsymbol{A}(i, i+h)_{(1 \longleftrightarrow n) / n}, \boldsymbol{T}, \boldsymbol{B}_{d}, \boldsymbol{K} \in \mathbb{R}^{n \times n}$.

Hereafter, $*$ denotes the symmetric item in a symmetric matrix.

Proof. Writing the closed-loop dynamics matrix $\boldsymbol{A}_{c}$ as follows:

$$
\left[\begin{array}{cccc}
a_{11} & a_{12} & \cdots & a_{1 n} \\
a_{21} & a_{22} & \cdots & a_{2 n} \\
& & \ddots & \\
a_{n 1} & a_{n 2} & \cdots & a_{n n}
\end{array}\right]-\left[\begin{array}{c}
b_{1} \\
b_{2} \\
\vdots \\
b_{n}
\end{array}\right]\left[\begin{array}{llll}
k_{1} & k_{2} & \cdots & k_{n}
\end{array}\right]<0,
$$

it is evident that $\boldsymbol{A}_{c}$ is a strictly Metzler matrix if all of its main diagonal elements are negative, i.e.,

$$
a_{i i}-b_{i} k_{i}<0 \quad \forall i=1,2, \ldots, n
$$

and all its of diagonal elements are positive, i.e.,

$$
a_{i j}-b_{i} k_{j}>0 \quad \forall i, j=1,2, \ldots, n, i \neq j .
$$

To solve by an LMI solver, LMIs have to be symmetric, and so (24) can be rewritten in the following diagonal matrix structure:

$$
\left[\begin{array}{llll}
a_{11} & & & \\
& a_{22} & & \\
& & \ddots & \\
& & & a_{n n}
\end{array}\right]
$$$$
-\left[\begin{array}{llll}
b_{1} & & & \\
& b_{2} & & \\
& & \ddots & \\
& & & b_{n}
\end{array}\right]\left[\begin{array}{llll}
k_{1} & & & \\
& k_{2} & & \\
& & \ddots & \\
& & & k_{n}
\end{array}\right] \prec 0,
$$

$$
\boldsymbol{A}(i, i)_{(1 \longleftrightarrow n)}-\boldsymbol{B}_{d} \boldsymbol{K} \prec 0,
$$

respectively, where $\boldsymbol{K}=\operatorname{diag}\left[\left\{k_{i}\right\}_{i=1, \ldots, n}\right]$ is the diagonal matrix variable.

Moreover, (26) implies

$$
\begin{aligned}
& {\left[\begin{array}{llll}
a_{11} & & & \\
& a_{22} & & \\
& & \ddots & \\
& & & a_{n n}
\end{array}\right]} \\
& \quad-\left[\begin{array}{llll}
k_{1} & & & \\
& k_{2} & & \\
& & \ddots & \\
& & & k_{n}
\end{array}\right]\left[\begin{array}{llll}
b_{1} & & & \\
& b_{2} & \\
& & \ddots & \\
& & & b_{n}
\end{array}\right]<0,
\end{aligned}
$$

That is, (27) is a symmetric matrix. Thus, exploiting (6), then (27) can be written as (15) using the notations (17) and (20), (21).

Rewriting (23) as

$$
\begin{gathered}
{\left[\begin{array}{ccccc}
a_{12} & a_{13} & \cdots & a_{1 n} & a_{11} \\
a_{22} & a_{23} & \cdots & a_{2 n} & a_{21} \\
& & \vdots & & \\
a_{n 2} & a_{n 3} & \cdots & a_{n n} & a_{n 1}
\end{array}\right]} \\
-\left[\begin{array}{c}
b_{1} \\
b_{2} \\
\vdots \\
b_{n}
\end{array}\right]\left[\begin{array}{lllll}
k_{2} & k_{3} & \cdots & k_{n} & k_{1}
\end{array}\right],
\end{gathered}
$$

it can set for the diagonal elements of (29)

$$
\begin{aligned}
& {\left[\begin{array}{llll}
a_{12} & & & \\
& a_{23} & & \\
& & \ddots & \\
& & & a_{n 1}
\end{array}\right]} \\
& \quad-\left[\begin{array}{llll}
b_{1} & & & \\
& b_{2} & \\
& & \ddots & \\
& & & b_{n}
\end{array}\right]\left[\begin{array}{llll}
k_{2} & & & \\
& k_{3} & \\
& & \ddots & \\
& & & k_{1}
\end{array}\right]>0,
\end{aligned}
$$

which leads to

$$
\boldsymbol{A}(i, i+1)_{(1 \longleftrightarrow n) / n}-\boldsymbol{B}_{d} \boldsymbol{K}_{c 1}>0,
$$

where $\boldsymbol{K}_{c 1}$ is the diagonal matrix $\boldsymbol{K}$ with one circular shift of its diagonal elements. It is evident that (31) is also a symmetric matrix. 
Repeating this procedure $h$-times, it can be obtained from (23) that

$$
\begin{gathered}
{\left[\begin{array}{cccccc}
a_{1,1+h} & \cdots & a_{1, n} & a_{1,1} & \cdots & a_{1, h} \\
a_{2, i+h} & \cdots & a_{2, n} & a_{2,1} & \cdots & a_{2, h} \\
\vdots & \ddots & \vdots & \vdots & \ddots & \vdots \\
a_{n, i+h} & \cdots & a_{n, n} & a_{n, 1} & \cdots & a_{n, h}
\end{array}\right]} \\
-\left[\begin{array}{c}
b_{1} \\
b_{2} \\
\vdots \\
b_{n}
\end{array}\right]\left[\begin{array}{llll}
k_{1+h} & k_{2+h} & \cdots & k_{h}
\end{array}\right],
\end{gathered}
$$

and, consequently,

$$
\begin{gathered}
{\left[\begin{array}{cccc}
a_{1,1+h} & & & \\
& a_{2,2+h} & & \\
& & \ddots & \\
& & & a_{n, h}
\end{array}\right]} \\
-\left[\begin{array}{cccc}
b_{1} & & & \\
& b_{2} & & \\
& & \ddots & \\
& & & b_{n}
\end{array}\right]\left[\begin{array}{llll}
k_{1+h} & & & \\
& k_{2+h} & & \\
& & \ddots & \\
& & & k_{h}
\end{array}\right]>0,
\end{gathered}
$$

which gives the symmetric matrix inequality

$$
\boldsymbol{A}(i, i+h)_{(1 \longleftrightarrow n) / n}-\boldsymbol{B}_{d} \boldsymbol{K}_{c, h}>0,
$$

where $\boldsymbol{K}_{c h}$ is the diagonal matrix $\boldsymbol{K}$ with $h$ circular shifts of its diagonal elements.

Using the permutation matrix $\boldsymbol{T}$ of the structure (58) [22] it can be easily verified that for $h=1,2, \ldots n-1$ it yields

$$
\boldsymbol{K}=\boldsymbol{T}^{h} \boldsymbol{K}_{c, h} \boldsymbol{T}^{-h}=\boldsymbol{T}^{h} \boldsymbol{K}_{c, h} \boldsymbol{T}^{h T} .
$$

Thus, premultiplying the right side by $\boldsymbol{T}^{h T}$ and postmultiplying the left side by $\boldsymbol{T}^{h}$ then (34) leads to

$$
\begin{array}{r}
\boldsymbol{T}^{h} \boldsymbol{A}(i, i+h)_{(1 \longleftrightarrow n) / n} \boldsymbol{T}^{h T}-\boldsymbol{T}^{h} \boldsymbol{B}_{d} \boldsymbol{T}^{h T} \boldsymbol{T}^{h} \boldsymbol{K}_{c, h} \boldsymbol{T}^{h T}>0, \\
\boldsymbol{T}^{h} \boldsymbol{A}(i, i+h)_{(1 \longleftrightarrow n) / n} \boldsymbol{T}^{h T}-\boldsymbol{T}^{h} \boldsymbol{B}_{d} \boldsymbol{T}^{h T} \boldsymbol{K}>0,
\end{array}
$$

respectively, and using (6), then (37) implies (16). This concludes the proof.

Theorem 13. The closed-loop system (12), (13) is stable strictly Metzlerian if system (9), (10) is strictly Metzlerian and there exist positive definite diagonal matrices $\boldsymbol{P}, \boldsymbol{R}, \boldsymbol{Q} \in \mathbb{R}^{n \times n}$ such that for $h=1,2, \ldots n-1$,

$$
\begin{aligned}
& \boldsymbol{A P}+\boldsymbol{P} \boldsymbol{A}^{T}-\boldsymbol{b r}^{T}-\boldsymbol{r} \boldsymbol{b}^{T}+\boldsymbol{Q} \prec 0, \\
& \frac{1}{2}\left(\boldsymbol{A}(i, i)_{(1 \longleftrightarrow n)} \boldsymbol{P}-\boldsymbol{B}_{d} \boldsymbol{R}\right)+(*) \prec 0, \\
& \frac{1}{2}\left(\boldsymbol{T}^{h} \boldsymbol{A}(i, i+h)_{(1 \longleftrightarrow n) / n} \boldsymbol{T}^{h T} \boldsymbol{P}-\boldsymbol{T}^{h} \boldsymbol{B}_{d} \boldsymbol{T}^{h T} \boldsymbol{R}\right)+(*) \\
& \quad>0, \\
& \boldsymbol{P}=\operatorname{diag}\left[\begin{array}{llll}
p_{1} & p_{2} & \cdots & p_{n}
\end{array}\right]>0, \\
& \boldsymbol{Q}=\operatorname{diag}\left[\begin{array}{llll}
q_{1} & q_{2} & \cdots & q_{n}
\end{array}\right]>0, \\
& \boldsymbol{R}=\operatorname{diag}\left[\begin{array}{llll}
r_{1} & r_{2} & \cdots & r_{n}
\end{array}\right]>0, \\
& \boldsymbol{r}^{T}=\left[\begin{array}{llll}
r_{1} & r_{2} & \cdots & r_{n}
\end{array}\right]=\boldsymbol{l}^{T} \boldsymbol{R}, \quad \boldsymbol{l}=\left[\begin{array}{llll}
1 & 1 & \cdots & 1
\end{array}\right]^{T},
\end{aligned}
$$

where the parameters are given in (18)-(22).

When the above conditions hold, the control law gain vector $\boldsymbol{k}^{T}$ is given as

$$
\boldsymbol{K}=\boldsymbol{R} \boldsymbol{P}^{-1}, \quad \boldsymbol{k}^{T}=\boldsymbol{l}^{T} \boldsymbol{K} .
$$

Proof. Inserting (14) into (4) gives

$$
\left(\boldsymbol{A}-\boldsymbol{b} \boldsymbol{k}^{T}\right) \boldsymbol{P}+\boldsymbol{P}\left(\boldsymbol{A}-\boldsymbol{b} \boldsymbol{k}^{T}\right)^{T}+\boldsymbol{Q} \prec 0 .
$$

Since with a positive diagonal matrix $\boldsymbol{P}$ it also yields

$$
\boldsymbol{A P}+\boldsymbol{P} \boldsymbol{A}^{T}-\boldsymbol{b} \boldsymbol{k}^{T} \boldsymbol{P}-\boldsymbol{P} \boldsymbol{k} \boldsymbol{b}^{T}+\boldsymbol{Q} \prec 0,
$$

using the notation

$$
\boldsymbol{r}^{T}=\boldsymbol{k}^{T} \boldsymbol{P}
$$

(47) implies (38).

Multiplying the right side of (27) by $\boldsymbol{P}$ gives

$$
\boldsymbol{A}(i, i)_{(1 \longleftrightarrow n)} \boldsymbol{P}-\boldsymbol{B}_{d} \boldsymbol{K} \boldsymbol{P} \prec 0,
$$

and with the notation

$$
\boldsymbol{R}=\boldsymbol{K P}
$$

(49) implies (39).

Multiplying the right side of (37) by $\boldsymbol{P}$ gives

$$
\boldsymbol{T}^{h} \boldsymbol{A}(i, i+h)_{(1 \longleftrightarrow n) / n} \boldsymbol{T}^{h T} \boldsymbol{P}-\boldsymbol{T}^{h} \boldsymbol{B}_{d} \boldsymbol{T}^{h T} \boldsymbol{K} \boldsymbol{P}>0
$$

and with the notation (50) then (51) implies (40). This concludes the proof.

Note that conditions (38)-(43) are all LMIs; that is, they are convex in the defined matrix variables.

Remark 14. It can be noted that the necessary diagonal matrix variable structure of $\boldsymbol{K}$, presented in Theorem 12, directly implies the diagonal matrix variable structures of $\boldsymbol{P}, \boldsymbol{R}$ in Theorem 13. Since, in the terms of the Krasovskii theorem [27], the matrices $\boldsymbol{Q}, \boldsymbol{U}$ in Proposition 7 can be zero matrices, this way can be also applied in (38), and in the inequalities we exploited the proposition properties in the following parts of the paper. 
3.2. Luenberger Observer Design. Considering the observable system (9), (10), the Luenberger observer is given as

$$
\begin{aligned}
& \dot{\boldsymbol{q}}_{e}(t)=\boldsymbol{A} \boldsymbol{q}_{e}(t)+\boldsymbol{b u}(t)+\boldsymbol{j}\left(y(t)-y_{e}(t)\right), \\
& y_{e}(t)=\boldsymbol{c}^{T} \boldsymbol{q}_{e}(t)
\end{aligned}
$$

and using (9), (10) and (52), (53) it yields

$$
\dot{\boldsymbol{e}}(t)=\left(\boldsymbol{A}-\boldsymbol{j} \boldsymbol{c}^{T}\right) \boldsymbol{e}(t)=\boldsymbol{A}_{e} \boldsymbol{e}(t),
$$

where

$$
\begin{aligned}
\boldsymbol{e}(t) & =\boldsymbol{q}(t)-\boldsymbol{q}_{e}(t), \\
\boldsymbol{A}_{e} & =\boldsymbol{A}-\boldsymbol{j} \boldsymbol{c}^{T},
\end{aligned}
$$

$\boldsymbol{q}_{e}(t) \in \mathbb{R}^{n}$ is the vector of the system state estimate, $y_{e}(t) \in$ $\mathbb{R}^{m}$ is the output variable estimate, and $\boldsymbol{j} \in \mathbb{R}^{n}$ is the observer gain vector.

Note that the observer has to be designed not only to be stable, but also to render the observer system matrix stable strictly Metzlerian.

Theorem 15. The Luenberger observer (52), (53) is strictly Metzlerian if system (9), (10) is strictly Metzlerian and there exists a positive definite diagonal matrix $\boldsymbol{J} \in \mathbb{R}^{n \times n}$ such that for $h=1,2, \ldots n-1$

$$
\begin{aligned}
& \frac{1}{2}\left(\boldsymbol{A}(i, i)_{(1 \longleftrightarrow n)}-J C_{d}\right)+(*) \prec 0 \\
& \frac{1}{2}\left(\boldsymbol{T}^{h} \boldsymbol{A}(i+h, i)_{(1 \longleftrightarrow n) / n} \boldsymbol{T}^{h T}-\boldsymbol{J} \boldsymbol{T}^{h} \boldsymbol{C}_{d} \boldsymbol{T}^{h T}\right)+(*) \\
& \quad>0 \\
& \boldsymbol{J}=\operatorname{diag}\left[\begin{array}{llll}
j_{1} & j_{2} & \cdots & j_{n}
\end{array}\right]>0
\end{aligned}
$$

where $\boldsymbol{T}$ is defined in (18),

$$
\begin{aligned}
& \boldsymbol{c}^{T}=\left[\begin{array}{llll}
c_{1} & c_{2} & \cdots & c_{n}
\end{array}\right] \\
& \boldsymbol{C}_{d}=\operatorname{diag}\left[\begin{array}{llll}
c_{1} & c_{2} & \cdots & c_{n}
\end{array}\right]=\operatorname{diag}\left[\left\{c_{i}\right\}_{i=1, \ldots, n}\right], \\
& \boldsymbol{A}(i, i)_{1 \longleftrightarrow n}=\operatorname{diag}\left[\begin{array}{llll}
a_{11} & a_{22} & \cdots & a_{n n}
\end{array}\right] \\
& =\operatorname{diag}\left[\left\{a_{i i}\right\}_{i=1, \ldots, n}\right] \text {, } \\
& \boldsymbol{A}(i+h, i)_{(1 \longleftrightarrow n) / n} \\
& =\operatorname{diag}\left[\begin{array}{lllllll}
a_{1+h, 1} & a_{2+h, 2} & \cdots & a_{n, n-h} & a_{1, n-h+1} & \cdots & a_{h, n}
\end{array}\right],
\end{aligned}
$$

while $\boldsymbol{A}(i, i)_{1 \longleftrightarrow n}, \boldsymbol{A}(i+h, i)_{(1 \longleftrightarrow n) / n}, \boldsymbol{T}, \boldsymbol{C}_{d}, \boldsymbol{J} \in \mathbb{R}^{n \times n}$.

Proof. Since the duality principle has to be applied on the structure (23), to avoid some misinterpretation the proof is given in partly full dual sense.

Noting down the observer matrix $\boldsymbol{A}_{e}$ structure (55) as

$$
\left[\begin{array}{cccc}
a_{11} & a_{12} & \cdots & a_{1 n} \\
a_{21} & a_{22} & \cdots & a_{2 n} \\
& & \ddots & \\
a_{n 1} & a_{n 2} & \cdots & a_{n n}
\end{array}\right]-\left[\begin{array}{c}
j_{1} \\
j_{2} \\
\vdots \\
j_{n}
\end{array}\right]\left[\begin{array}{llll}
c_{1} & c_{2} & \cdots & c_{n}
\end{array}\right] \prec 0,
$$

it is evident that $\boldsymbol{A}_{e}$ is a strictly Metzler matrix if

$$
\begin{aligned}
a_{i i}-j_{i} c_{i}<0 & \forall i=1,2, \ldots, n, \\
a_{i j}-j_{i} c_{j}>0 & \forall i, j=1,2, \ldots, n, i \neq j .
\end{aligned}
$$

Writing (64) in the diagonal matrix structure

$$
\begin{gathered}
{\left[\begin{array}{llll}
a_{11} & & & \\
& a_{22} & & \\
& & \ddots & \\
& & & a_{n n}
\end{array}\right]} \\
-\left[\begin{array}{llll}
j_{1} & & \\
& j_{2} & \\
& & \ddots & \\
& & & j_{n}
\end{array}\right]\left[\begin{array}{llll}
c_{1} & & \\
& c_{2} & & \\
& & \ddots & \\
& & & c_{n}
\end{array}\right] \prec 0, \\
\boldsymbol{A}(i, i)_{(1 \longleftrightarrow n)}-\boldsymbol{J C}_{d} \prec 0, \\
\end{gathered}
$$

respectively, where $\boldsymbol{J}=\operatorname{diag}\left[\left\{j_{i}\right\}_{i=1, \ldots, n}\right]$ is the diagonal matrix variable, it is evident that such structure is symmetric and (67) can be written as (56) using notations (60), (61).

Overwriting inequality (63) as

$$
\left[\begin{array}{cccc}
a_{21} & a_{22} & \cdots & a_{2 n} \\
a_{31} & a_{32} & \cdots & a_{3 n} \\
& & \vdots & \\
a_{n 1} & a_{n 2} & \cdots & a_{n n} \\
a_{11} & a_{12} & \cdots & a_{1 n}
\end{array}\right]-\left[\begin{array}{c}
j_{2} \\
j_{3} \\
\vdots \\
j_{n} \\
j_{1}
\end{array}\right]\left[\begin{array}{llll}
c_{1} & c_{2} & \cdots & c_{n}
\end{array}\right]
$$

it has to be for the diagonal elements of (68)

$$
\left[\begin{array}{cccc}
a_{21} & & & \\
& a_{32} & & \\
& & \ddots & \\
& & & a_{1 n}
\end{array}\right]
$$

$$
-\left[\begin{array}{cccc}
j_{2} & & & \\
& j_{3} & & \\
& & \ddots & \\
& & & j_{1}
\end{array}\right]\left[\begin{array}{llll}
c_{1} & & & \\
& c_{2} & & \\
& & \ddots & \\
& & & c_{n}
\end{array}\right]>0,
$$

which leads to the symmetric inequality

$$
\boldsymbol{A}(i+1, i)_{(1 \longleftrightarrow n) / n}-\boldsymbol{J}_{c 1} C_{d}>0,
$$

where $\boldsymbol{J}_{c 1}$ is the diagonal matrix $\boldsymbol{J}$ with one circular shift of its diagonal elements. 
Repeating this procedure $h$-times, it can obtain the following:

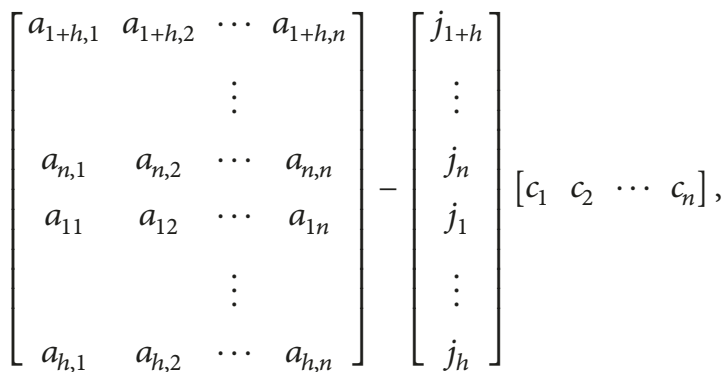

$$
\begin{aligned}
& {\left[\begin{array}{cccc}
a_{1+h, 1} & & & \\
& a_{2+h, 2} & & \\
& & \ddots & \\
& & & a_{h, n}
\end{array}\right]} \\
& -\left[\begin{array}{cccc}
j_{1+h} & & & \\
& j_{2+h} & & \\
& & \ddots & \\
& & & j_{h}
\end{array}\right]\left[\begin{array}{llll}
c_{1} & & & \\
& c_{2} & & \\
& & \ddots & \\
& & & c_{n}
\end{array}\right]>0 \text {, }
\end{aligned}
$$

respectively. Thus, analogously, (72) results in

$$
\boldsymbol{A}(i+h, i)_{(1 \longleftrightarrow n) / n}-\boldsymbol{J}_{c, h} \boldsymbol{C}_{d}>0 .
$$

Using the permutation matrix $\boldsymbol{T}$ of the structure (58) it yields for $h=1,2, \ldots n-1$ that

$$
\boldsymbol{J}=\boldsymbol{T}^{h} \boldsymbol{J}_{c, h} \boldsymbol{T}^{-h}=\boldsymbol{T}^{h} \boldsymbol{J}_{c, h} \boldsymbol{T}^{h T}
$$

and premultiplying the left side by $\boldsymbol{T}^{h}$ and postmultiplying the right side by $\boldsymbol{T}^{h T}$ then the symmetric structure (73)

$$
\begin{aligned}
\boldsymbol{T}^{h} \boldsymbol{A}(i+h, i)_{(1 \longleftrightarrow n) / n} \boldsymbol{T}^{h T}-\boldsymbol{T}^{h} \boldsymbol{J}_{c, h} \boldsymbol{T}^{h T} \boldsymbol{T}^{h} \boldsymbol{C}_{d} \boldsymbol{T}^{h T}>0 \\
\boldsymbol{T}^{h} \boldsymbol{A}(i+h, i)_{(1 \longleftrightarrow n) / n} \boldsymbol{T}^{h T}-\boldsymbol{J} \boldsymbol{T}^{h} \boldsymbol{C}_{d} \boldsymbol{T}^{h T}>0
\end{aligned}
$$

respectively, and using (6), then (76) implies (57). This concludes the proof.

Theorem 16. The Luenberger observer (52), (53) is stable strictly Metzlerian if system (9), (10) is strictly Metzlerian and there exist positive definite diagonal matrices $\boldsymbol{U}, \boldsymbol{V}, \boldsymbol{W} \in \mathbb{R}^{n \times n}$ such that for $h=1,2, \ldots n-1$

$$
\begin{aligned}
& \boldsymbol{V} \boldsymbol{A}+\boldsymbol{A}^{T} \boldsymbol{V}-\boldsymbol{w} \boldsymbol{c}^{T}-\boldsymbol{c} \boldsymbol{w}^{T}+\boldsymbol{U} \prec 0, \\
& \frac{1}{2}\left(\boldsymbol{V} \boldsymbol{A}(i, i)_{(1 \longleftrightarrow n)}-\boldsymbol{W} \boldsymbol{C}_{d}\right)+(*) \prec 0, \\
& \frac{1}{2}\left(\boldsymbol{V} \boldsymbol{T}^{h} \boldsymbol{A}(i+h, i)_{(1 \longleftrightarrow n) / n} \boldsymbol{T}^{h T}-\boldsymbol{W} \boldsymbol{T}^{h} \boldsymbol{C}_{d} \boldsymbol{T}^{h T}\right)+(*) \\
& \quad>0, \\
& \boldsymbol{V}=\operatorname{diag}\left[\begin{array}{llll}
v_{1} & v_{2} & \cdots & v_{n}
\end{array}\right]>0, \\
& \boldsymbol{W}=\operatorname{diag}\left[\begin{array}{llll}
w_{1} & w_{2} & \cdots & w_{n}
\end{array}\right]>0, \\
& \boldsymbol{U}=\operatorname{diag}\left[\begin{array}{llll}
u_{1} & u_{2} & \cdots & u_{n}
\end{array}\right]>0, \\
& \boldsymbol{w}^{T}=\left[\begin{array}{llll}
w_{1} & w_{2} & \cdots & w_{n}
\end{array}\right]=\boldsymbol{l}^{T} \boldsymbol{W}, \quad \boldsymbol{l}=\left[\begin{array}{llll}
1 & 1 & \cdots & 1
\end{array}\right]^{T},
\end{aligned}
$$

where the parameters are given in (18), (59)-(62).

When the above conditions hold, the observer gain vector $\mathbf{j}$ is given as

$$
\boldsymbol{J}=\boldsymbol{V}^{-1} \boldsymbol{W}, \quad \boldsymbol{j}=\boldsymbol{J} \boldsymbol{}
$$

Proof. Inserting (55) into (5) gives for a positive definite diagonal matrix $\boldsymbol{V}$ that

$$
\begin{gathered}
\boldsymbol{V}\left(\boldsymbol{A}-\boldsymbol{j} \boldsymbol{c}^{T}\right)+\left(\boldsymbol{A}-\boldsymbol{j} \boldsymbol{c}^{T}\right)^{T} \boldsymbol{V}+\boldsymbol{U} \prec 0 \\
\boldsymbol{V} \boldsymbol{A}+\boldsymbol{A}^{T} \boldsymbol{V}-\boldsymbol{V} \boldsymbol{j} \boldsymbol{c}^{T}-\boldsymbol{c} \boldsymbol{j}^{T} \boldsymbol{V}+\boldsymbol{U} \prec 0
\end{gathered}
$$

respectively. Therefore, using the notation

$$
\boldsymbol{w}^{T}=\boldsymbol{j}^{T} \boldsymbol{V},
$$

(86) implies (77)

Multiplying the left side of (67) by $\boldsymbol{V}$ gives

$$
\boldsymbol{V A}(i, i)_{(1 \longleftrightarrow n)}-\boldsymbol{V J} \boldsymbol{C}_{d} \prec 0,
$$

and with the notation

$$
\boldsymbol{W}=\boldsymbol{V} \boldsymbol{J}
$$

(88) implies (78).

Multiplying the left side of (76) by $\boldsymbol{V}$ gives

$$
\boldsymbol{V} \boldsymbol{T}^{h} \boldsymbol{A}(i, i+h)_{(1 \longleftrightarrow n) / n} \boldsymbol{T}^{h T}-\boldsymbol{V} \boldsymbol{J} \boldsymbol{T}^{h} \boldsymbol{C}_{d} \boldsymbol{T}^{h T}>0
$$

and with the notation (89) then (90) implies (79). This concludes the proof.

Also conditions (77)-(82) are all LMIs that is they are convex in the defined matrix variables. 
3.3. Supporting Matrix Structures. To simplify obtaining $\boldsymbol{A}(i, i+h)_{(1 \longleftrightarrow n) / n}$ or $\boldsymbol{A}(i+h, i)_{(1 \longleftrightarrow n) / n}$, it is possible to construct the following matrices:

$$
\begin{aligned}
& A^{\circ}=\left[\begin{array}{ll}
A & A
\end{array}\right] \\
& =\left[\begin{array}{cccccccc}
a_{11} & a_{12} & \cdots & a_{1 n} & a_{11} & a_{12} & \cdots & a_{1 n} \\
a_{21} & a_{22} & \cdots & a_{2 n} & a_{21} & a_{22} & \cdots & a_{2 n} \\
\vdots & & \ddots & \vdots & \ddots & \\
a_{11} & a_{12} & \cdots & a_{1 n} & a_{11} & a_{12} & \cdots & a_{1 n}
\end{array}\right] \text {, } \\
& \boldsymbol{A}^{\diamond}=\left[\begin{array}{c}
\boldsymbol{A} \\
\boldsymbol{A}
\end{array}\right]=\left[\begin{array}{cccc}
a_{11} & a_{12} & \cdots & a_{1 n} \\
a_{21} & a_{22} & \cdots & a_{2 n} \\
\vdots & & \ddots & \\
a_{n 1} & a_{n 2} & \cdots & a_{n n} \\
a_{11} & a_{12} & \cdots & a_{1 n} \\
a_{21} & a_{22} & \cdots & a_{2 n} \\
\vdots & & \ddots & \\
a_{n 1} & a_{n 2} & \cdots & a_{n n}
\end{array}\right] \text {, }
\end{aligned}
$$

respectively.

Then, using the set of $n-1$ upper subdiagonals of dimension of $n$, the matrices $\boldsymbol{A}(i, i+h)_{(1 \longleftrightarrow n) / n}$ can be sequentially constructed for $h=1,2, \ldots n-1$ from (91) and, exploiting the set of $n-1$ lower subdiagonals of dimension of $n$, the matrices $\boldsymbol{A}(i+h, i)_{(1 \longleftrightarrow n) / n}$ can be sequentially constructed for $h=1,2, \ldots n-1$ reflecting (92).

\section{MIMO Strictly Metzlerian Systems}

Linear multiple-input, multiple-output (MIMO) continuoustime closed-loop dynamical systems obtained from the controllable system (1), (2) by using the state control law

$$
\boldsymbol{u}(t)=-\boldsymbol{K} \boldsymbol{q}(t), \quad \boldsymbol{K} \in \mathbb{R}^{r \times n}
$$

are described by the state-space equations

$$
\begin{aligned}
& \dot{\boldsymbol{q}}(t)=(\boldsymbol{A}-\boldsymbol{B} \boldsymbol{K}) \boldsymbol{q}(t)=\boldsymbol{A}_{c} \boldsymbol{q}(t), \\
& \boldsymbol{y}(t)=\boldsymbol{C} \boldsymbol{q}(t),
\end{aligned}
$$

where

$$
A_{c}=A-B K
$$

The MIMO Luenberger observer, associated with the observable system (1), (2), is given as

$$
\begin{aligned}
\dot{\boldsymbol{q}}_{e}(t) & =\boldsymbol{A} \boldsymbol{q}_{e}(t)+\boldsymbol{B} \boldsymbol{u}(t)+\boldsymbol{J}\left(\boldsymbol{y}(t)-\boldsymbol{y}_{e}(t)\right), \\
\boldsymbol{y}_{e}(t) & =\boldsymbol{C} \boldsymbol{q}_{e}(t), \\
\dot{\boldsymbol{e}}(t) & =(\boldsymbol{A}-\boldsymbol{J C}) \boldsymbol{e}(t)=\boldsymbol{A}_{e} \boldsymbol{e}(t),
\end{aligned}
$$

where

$$
\begin{aligned}
\boldsymbol{e}(t) & =\boldsymbol{q}(t)-\boldsymbol{q}_{e}(t), \\
\boldsymbol{A}_{e} & =\boldsymbol{A}-\boldsymbol{J} \boldsymbol{C} .
\end{aligned}
$$

Naturally, if $\boldsymbol{A} \in \mathbb{R}^{n \times n}$ is Metzler matrix, and $\boldsymbol{B} \in$ $\mathbb{R}_{+}^{n \times r}, \boldsymbol{C} \in \mathbb{R}_{+}^{m \times n}$ are nonnegative matrices, system (1), (2) is Metzlerian system. Thus, it is necessary to render the closedloop system matrix $\boldsymbol{A}_{c}$ and observer system matrix $\boldsymbol{A}_{e}$ as stable strictly Metzler ones.

Theorem 17. The closed-loop system (94), (95) is strictly Metzlerian if system (1), (2) is strictly Metzlerian and there exist positive definite diagonal matrices $\boldsymbol{K}_{k} \in \mathbb{R}^{n \times n}$ such that for $h=1,2, \ldots n-1, k=1,2 \ldots, r$,

$$
\begin{aligned}
& \frac{1}{2}\left(\boldsymbol{A}(i, i)_{1 \longleftrightarrow n}-\sum_{k=1}^{r} \boldsymbol{B}_{d k} \boldsymbol{K}_{k}\right)+(*) \prec 0, \\
& \frac{1}{2}\left(\boldsymbol{T}^{h} \boldsymbol{A}(i, i+h)_{(1 \longleftrightarrow n) / n} \boldsymbol{T}^{h T}-\sum_{k=1}^{r} \boldsymbol{T}^{h} \boldsymbol{B}_{d k} \boldsymbol{T}^{h T} \boldsymbol{K}_{k}\right) \\
& \quad+(*) \succ 0, \\
& \boldsymbol{K}_{k}=\operatorname{diag}\left[\begin{array}{llll}
k_{k 1} & k_{k 2} & \cdots & k_{k n}
\end{array}\right]>0,
\end{aligned}
$$

where $\boldsymbol{T}$ is defined in (18) and $\boldsymbol{A}(i, i)_{1 \longleftrightarrow n}, \boldsymbol{A}(i, i+h)_{(1 \longleftrightarrow n) / n}$ are introduced in (21), (22), respectively,

$$
\begin{aligned}
& \boldsymbol{B}=\left[\begin{array}{cccc}
b_{11} & b_{12} & \cdots & b_{1 r} \\
b_{21} & b_{22} & \cdots & b_{2 r} \\
& & \vdots & \\
& & & \\
b_{n 1} & b_{n 2} & \cdots & b_{n r}
\end{array}\right] \text {, } \\
& \boldsymbol{B}_{d k}=\operatorname{diag}\left[b_{1 k} \quad b_{2 k} \cdots b_{n k}\right]=\operatorname{diag}\left[\left\{b_{i k}\right\}_{i=1, \ldots, n}\right] \text {, }
\end{aligned}
$$

while $\boldsymbol{B}_{d k}, \boldsymbol{K}_{k} \in \mathbb{R}^{n \times n}$.

Proof. In analogy with (24) it is evident that $\boldsymbol{A}_{c}$ is Metzler matrix if all of its main diagonal elements satisfy the conditions

$$
a_{i i}-\sum_{k=1}^{r} b_{k i} k_{k i}<0 \quad \forall i=1,2, \ldots, n
$$

and all its of diagonal elements satisfy the inequalities

$$
a_{i j}-\sum_{k=1}^{r} b_{k i} k_{k j}>0 \quad \forall i, j=1,2, \ldots, n, i \neq j .
$$

Thus, using the above notations and (103), (105), then (106) implies

$$
\boldsymbol{A}(i, i)_{(1 \longleftrightarrow n)}-\sum_{k=1}^{r} \boldsymbol{B}_{d k} \boldsymbol{K}_{k}>0
$$

and, consequently, (108) implies (101). 
In the same way, (107) can be interpreted as

$$
\boldsymbol{T}^{h} \boldsymbol{A}(i, i+h)_{(1 \longleftrightarrow n) / n} \boldsymbol{T}^{h T}-\sum_{k=1}^{r} \boldsymbol{T}^{h} \boldsymbol{B}_{d k} \boldsymbol{T}^{h T} \boldsymbol{K}_{k}>0
$$

and (109) implies (102). This concludes the proof.

Theorem 18. The closed-loop system (12), (13) is stable strictly Metzlerian if system (1), (2) is strictly Metzlerian and there exist positive definite diagonal matrices $\boldsymbol{P}, \boldsymbol{R}_{k}, \mathbf{Q} \in \mathbb{R}^{n \times n}$ such that for $h=1,2, \ldots n-1, k=1,2, \ldots r$,

$$
\begin{aligned}
& \boldsymbol{A P}+\boldsymbol{P} \boldsymbol{A}^{T}-\sum_{k=1}^{r}\left(\boldsymbol{b}_{k} \boldsymbol{r}_{k}^{T}+\boldsymbol{r}_{k} \boldsymbol{b}_{k}^{T}\right)+\boldsymbol{Q} \prec 0, \\
& \frac{1}{2}\left(\boldsymbol{A}(i, i)_{(1 \longleftrightarrow n)} \boldsymbol{P}-\sum_{k=1}^{r} \boldsymbol{B}_{d k} \boldsymbol{R}_{k}\right)+(*) \prec 0, \\
& \frac{1}{2}\left(\boldsymbol{T}^{h} \boldsymbol{A}(i, i+h)_{(1 \longleftrightarrow n) / n} \boldsymbol{T}^{h T} \boldsymbol{P}-\sum_{k=1}^{r} \boldsymbol{T}^{h} \boldsymbol{B}_{d k} \boldsymbol{T}^{h T} \boldsymbol{R}_{k}\right) \\
& +(*)>0, \\
& \boldsymbol{P}=\operatorname{diag}\left[\begin{array}{llll}
p_{1} & p_{2} & \cdots & p_{n}
\end{array}\right]>0, \\
& \mathbf{Q}=\operatorname{diag}\left[\begin{array}{llll}
q_{1} & q_{2} & \cdots & q_{n}
\end{array}\right]>0, \\
& \boldsymbol{R}_{k}=\operatorname{diag}\left[\begin{array}{llll}
r_{k 1} & r_{k 2} & \cdots & r_{k n}
\end{array}\right]>0, \\
& \boldsymbol{r}_{k}^{T}=\left[\begin{array}{llll}
r_{k 1} & r_{k 2} & \cdots & r_{k n}
\end{array}\right]=\boldsymbol{l}^{T} \boldsymbol{R}_{k}, \quad \boldsymbol{l}=\left[\begin{array}{llll}
1 & 1 & \cdots & 1
\end{array}\right]^{T},
\end{aligned}
$$

where $\boldsymbol{b}_{k}^{T}=\boldsymbol{l}^{T} \boldsymbol{B}_{d k}$ and the remaining parameters are given in (18), (21), and (22).

When the above conditions hold, the control gain matrix $\boldsymbol{K}$ is given as

$$
\begin{gathered}
\boldsymbol{K}_{k}=\boldsymbol{R}_{k} \boldsymbol{P}^{-1}, \\
\boldsymbol{k}_{k}^{T}=\boldsymbol{l}^{T} \boldsymbol{K}_{k}, \\
\boldsymbol{K}=\left[\begin{array}{c}
\boldsymbol{k}_{1}^{T} \\
\vdots \\
\boldsymbol{k}_{r}^{T}
\end{array}\right] .
\end{gathered}
$$

Proof. Inserting (96) into (4) gives

$$
\begin{array}{r}
(\boldsymbol{A}-\boldsymbol{B} \boldsymbol{K}) \boldsymbol{P}+\boldsymbol{P}(\boldsymbol{A}-\boldsymbol{B} \boldsymbol{K})^{T}+\boldsymbol{Q} \prec 0 . \\
\left(\boldsymbol{A}-\sum_{k=1}^{r} \boldsymbol{b}_{k} \boldsymbol{k}_{k}^{T}\right) \boldsymbol{P}+\boldsymbol{P}\left(\boldsymbol{A}-\sum_{k=1}^{r} \boldsymbol{b}_{k} \boldsymbol{k}^{T}\right)^{T}+\boldsymbol{Q} \prec 0 .
\end{array}
$$

respectively, and using the notation

$$
\boldsymbol{r}_{k}^{T}=\boldsymbol{k}_{k}^{T} \boldsymbol{P}
$$

(119) implies (110).
Since multiplying the right side of (108), (109) by $\boldsymbol{P}$ leads to

$$
\begin{array}{r}
\boldsymbol{A}(i, i)_{(1 \longleftrightarrow n)} \boldsymbol{P}-\sum_{k=1}^{r} \boldsymbol{B}_{d k} \boldsymbol{K}_{k} \boldsymbol{P} \prec 0, \\
\boldsymbol{T}^{h} \boldsymbol{A}(i, i+h)_{(1 \longleftrightarrow n) / n} \boldsymbol{T}^{h T} \boldsymbol{P}-\sum_{k=1}^{r} \boldsymbol{T}^{h} \boldsymbol{B}_{d k} \boldsymbol{T}^{h T} \boldsymbol{K}_{k} \boldsymbol{P}>0,
\end{array}
$$

then, with the notation

$$
\boldsymbol{R}_{k}=\boldsymbol{K}_{k} \boldsymbol{P}
$$

(121) and (122) imply (111), (112), respectively. This concludes the proof.

Remark 19. The point was finding conditions for the synthesis of the state control law to stabilize positive systems that would be sufficiently general. The stabilizability is bound to a particular system pair $(\boldsymbol{A}, \boldsymbol{B})$ and the strictly Metzler matrix $\boldsymbol{A}$ does not a priori generate any boundaries on elements of the nonnegative matrix $\boldsymbol{B}$ to obtain that the matrix of closedloop dynamics $\boldsymbol{A}_{c}=\boldsymbol{A}-\boldsymbol{B} \boldsymbol{K}$ is (strictly) Metzler matrix if $\boldsymbol{K}$ is positive matrix.

If the Metzler matrix element $a_{i j}=0, i \neq j$ it follows for given $i$ from condition (107) that all elements of row $i$ of the matrix $\boldsymbol{B}$ must be zero. With such a boundary on the structure of the matrix $\boldsymbol{B}$, the positive gain matrix $\boldsymbol{K}$, if exists, can be designed according to Theorem 18.

The second way is to design $K$ as nonnegative matrix. In this case, if $a_{i j}=0, i \neq j$, it is necessary to define the diagonal matrices $\boldsymbol{R}_{k}$ in (115) as structured matrix variables, whose $r_{k j}$ elements are zero for given $j$, and then the nonnegative gain matrix $\boldsymbol{K}$, if exists, can be designed according to Theorem 18 . Potentially, both ways can be combined.

Analogously the above-mentioned approaches can be applied in Luenberger observer synthesis for Metzlerian systems.

Theorem 20. The Luenberger observer (97), (98) is strictly Metzlerian if system (1), (2) is strictly Metzlerian and there exist positive definite diagonal matrices $\boldsymbol{J}_{k} \in \mathbb{R}^{n \times n}$ such that for $h=1,2, \ldots n-1, k=1,2, \ldots m$,

$$
\begin{aligned}
& \frac{1}{2}\left(\boldsymbol{A}(i, i)_{1 \longleftrightarrow n}-\sum_{k=1}^{m} \boldsymbol{J}_{k} \boldsymbol{C}_{d k}\right)+(*) \prec 0, \\
& \frac{1}{2}\left(\boldsymbol{T}^{h} \boldsymbol{A}(i+h, i)_{(1 \longleftrightarrow n) / n} \boldsymbol{T}^{h T}-\sum_{k=1}^{m} \boldsymbol{J}_{k} \boldsymbol{T}^{h} \boldsymbol{C}_{k} \boldsymbol{T}^{h T}\right) \\
& \quad+(*) \succ 0, \\
& \boldsymbol{J}_{k}=\operatorname{diag}\left[\begin{array}{llll}
j_{1 k} & j_{2 k} & \cdots & j_{n k}
\end{array}\right]>0,
\end{aligned}
$$


where $\boldsymbol{T}$ is defined in (18), $\boldsymbol{A}(i, i)_{1 \longleftrightarrow n}, \boldsymbol{A}(i+h, i)_{(1 \longleftrightarrow n) / n}$ is introduced in (61), (62), respectively,

$$
\begin{aligned}
C & =\left[\begin{array}{cccc}
c_{11} & c_{12} & \cdots & c_{1 n} \\
c_{21} & c_{22} & \cdots & c_{2 n} \\
& & \vdots & \\
c_{m 1} & c_{m 2} & \cdots & c_{m n}
\end{array}\right], \\
\boldsymbol{C}_{d k} & =\operatorname{diag}\left[\begin{array}{llll}
c_{k 1} & c_{k 2} & \cdots & c_{k n}
\end{array}\right], \quad k=1,2 \ldots, m,
\end{aligned}
$$

while $\boldsymbol{C}_{d k} \in \mathbb{R}^{n \times n}$.

Proof. In analogy with (63) it is evident that $\boldsymbol{A}_{e}$ is Metzler matrix if

$$
\begin{aligned}
& a_{i i}-\sum_{k=1}^{m} j_{k i} c_{k i}<0 \quad \forall i=1,2, \ldots, n, \\
& a_{i j}-\sum_{k=1}^{m} j_{k i} c_{k j}>0 \quad \forall i, j=1,2, \ldots, n, i \neq j .
\end{aligned}
$$

Thus, using the above notations and (126), (127) then (129) implies

$$
\boldsymbol{A}(i, i)_{(1 \longleftrightarrow n)}-\sum_{k=1}^{m} \boldsymbol{J}_{k} \boldsymbol{C}_{d k}>0
$$

and, consequently, (131) implies (124).

In the same way, (130) can be interpreted as

$$
\boldsymbol{T}^{h} \boldsymbol{A}(i, i+h)_{(1 \longleftrightarrow n) / n} \boldsymbol{T}^{h T}-\sum_{k=1}^{m} \boldsymbol{J}_{k} \boldsymbol{T}^{h} \boldsymbol{C}_{d k} \boldsymbol{T}^{h T}>0
$$

and (132) implies (125). This concludes the proof.

Theorem 21. The Luenberger observer (97), (98) is stable strictly Metzlerian if system (1), (2) is strictly Metzlerian and there exists a positive definite diagonal matrices $\boldsymbol{U}, \boldsymbol{V}, \boldsymbol{W}_{k} \in$ $\mathbb{R}^{n \times n}$ such that for $h=1,2, \ldots n-1, k=1,2, \ldots m$,

$$
\begin{aligned}
& \boldsymbol{V} \boldsymbol{A}+\boldsymbol{A}^{T} \boldsymbol{V}-\sum_{k=1}^{m}\left(\boldsymbol{w}_{k} \boldsymbol{c}_{k}^{T}+\boldsymbol{c}_{k} \boldsymbol{w}_{k}^{T}\right)+\boldsymbol{U} \prec 0, \\
& \frac{1}{2}\left(\boldsymbol{V A}(i, i)_{(1 \longleftrightarrow n)}-\sum_{k=1}^{m} \boldsymbol{W}_{k} \boldsymbol{C}_{d k}\right)+(*) \prec 0, \\
& \frac{1}{2}\left(\boldsymbol{V} \boldsymbol{T}^{h} \boldsymbol{A}(i+h, i)_{(1 \longleftrightarrow n) / n} \boldsymbol{T}^{h T}\right. \\
& \left.-\sum_{k=1}^{m} \boldsymbol{W}_{k} \boldsymbol{T}^{h} \boldsymbol{C}_{d k} \boldsymbol{T}^{h T}\right)+(*)>0, \\
& \boldsymbol{V}=\operatorname{diag}\left[\begin{array}{llll}
v_{1} & v_{2} & \cdots & v_{n}
\end{array}\right]>0, \\
& \boldsymbol{U}=\operatorname{diag}\left[\begin{array}{llll}
u_{1} & u_{2} & \cdots & u_{n}
\end{array}\right]>0, \\
& \boldsymbol{W}_{k}=\operatorname{diag}\left[\begin{array}{llll}
w_{k 1} & w_{k 2} & \cdots & w_{k n}
\end{array}\right]>0, \\
& \boldsymbol{w}_{k}^{T}=\left[\begin{array}{llll}
w_{k 1} & w_{k 2} & \cdots & w_{k n}
\end{array}\right]=\boldsymbol{l}^{T} \boldsymbol{W}_{k}, \\
& \boldsymbol{l}=\left[\begin{array}{llll}
1 & 1 & \cdots & 1
\end{array}\right]^{T},
\end{aligned}
$$

where $\boldsymbol{c}_{k}^{T}=\boldsymbol{l}^{T} \boldsymbol{C}_{d k}$ and the remaining parameters are given in (18), (61), and (62).

When the above conditions hold, the observer gain matrix $J$ is given as

$$
\begin{aligned}
\boldsymbol{J}_{k} & =\boldsymbol{V}^{-1} \boldsymbol{W}_{k}, \\
\boldsymbol{j}_{k} & =\boldsymbol{J}_{k} \boldsymbol{l}, \\
\boldsymbol{J} & =\left[\begin{array}{lll}
\boldsymbol{j}_{1} & \cdots & \boldsymbol{j}_{m}
\end{array}\right] .
\end{aligned}
$$

Proof. Inserting (100) into (5) gives

$$
\begin{array}{r}
\boldsymbol{V}(\boldsymbol{A}-\boldsymbol{J} \boldsymbol{C})+(\boldsymbol{A}-\boldsymbol{J} \boldsymbol{C})^{T} \boldsymbol{V}+\boldsymbol{U} \prec 0, \\
\boldsymbol{V}\left(\boldsymbol{A}-\sum_{k=1}^{m} \boldsymbol{j}_{k} \boldsymbol{c}_{k}^{T}\right)+\left(\boldsymbol{A}-\sum_{k=1}^{m} \boldsymbol{j}_{k} \boldsymbol{c}_{k}^{T}\right)^{T} \boldsymbol{V}+\boldsymbol{U} \prec 0,
\end{array}
$$

respectively. Therefore, using the notation

$$
\boldsymbol{w}_{k}^{T}=\boldsymbol{j}_{k}^{T} \boldsymbol{V}
$$

(142) implies (133).

Multiplying the left side of (131) and (132) by $\boldsymbol{V}$ gives

$$
\boldsymbol{V A}(i, i)_{(1 \longleftrightarrow n)}-\sum_{k=1}^{m} \boldsymbol{V} \boldsymbol{J}_{k} \boldsymbol{C}_{d k} \prec 0,
$$

$$
\boldsymbol{V} \boldsymbol{T}^{h} \boldsymbol{A}(i, i+h)_{(1 \longleftrightarrow n) / n} \boldsymbol{T}^{h T}-\sum_{k=1}^{m} \boldsymbol{V} \boldsymbol{J}_{k} \boldsymbol{T}^{h} \boldsymbol{C}_{d k} \boldsymbol{T}^{h T}>0,
$$


respectively, and with the notation

$$
\boldsymbol{W}_{k}=\boldsymbol{V} \boldsymbol{J}_{k}
$$

(144), (145) imply (134), (135), respectively. This concludes the proof.

Remark 22. If the boundary conditions can be formulated as quadratic boundaries (e.g., LQ constraints on state and inputs, $H_{\infty}$-norm, $D$-stability circle area, etc.), they can be included in the presented design conditions for positive systems because they only modify the structure of the LMIs (110), (133), forcing the system stability or add new LMSs.

\section{Illustrative Example}

The considered strictly Metzlerian system is represented by the model (1), (2) with the parameters

$$
\begin{aligned}
& \boldsymbol{A}=\left[\begin{array}{cccc}
-3.3800 & 0.2080 & 6.7150 & 5.6760 \\
0.5810 & -4.2900 & 2.0500 & 0.6750 \\
1.0670 & 4.2730 & -6.6540 & 5.8930 \\
0.0480 & 2.2730 & 1.3430 & -2.1040
\end{array}\right] \text {, } \\
& \boldsymbol{B}=\left[\begin{array}{ll}
0.0400 & 0.0189 \\
0.0568 & 0.0203 \\
0.0114 & 0.0315 \\
0.0114 & 0.0170
\end{array}\right] \text {, } \\
& C=\left[\begin{array}{llll}
4 & 0 & 1 & 0 \\
0 & 0 & 0 & 1
\end{array}\right] \text {. }
\end{aligned}
$$

It is possible to verify that the Metzler matrix $\boldsymbol{A}$ is unstable with the eigenvalue spectrum

$$
\rho(A)=\{1.9761,-9.4392,-4.4824 \pm 1.2499 \mathrm{i}\} .
$$

To solve the stabilization task, the auxiliary parameters are constructed as

$$
\boldsymbol{T}=\left[\begin{array}{llll}
0 & 0 & 0 & 1 \\
1 & 0 & 0 & 0 \\
0 & 1 & 0 & 0 \\
0 & 0 & 1 & 0
\end{array}\right]
$$

$$
\begin{aligned}
& \boldsymbol{A}(i, i)_{(1 \longleftrightarrow 4)} \\
& =\left[\begin{array}{lrrr}
-3.3800 & & & \\
& -4.2900 & & \\
& & -6.6540 & \\
& & & -2.1040
\end{array}\right]
\end{aligned}
$$

$$
\boldsymbol{A}(i, i+1)_{(1 \longleftrightarrow 4) / 4}
$$

$$
=\left[\begin{array}{llll}
0.2080 & & & \\
& 2.0500 & & \\
& & 5.8930 & \\
& & & 0.0480
\end{array}\right] \text {, }
$$

$\boldsymbol{A}(i, i+2)_{(1 \longleftrightarrow 4) / 4}$

$$
=\left[\begin{array}{llll}
6.7150 & & & \\
& 0.6750 & & \\
& & 1.0670 & \\
& & & 2.2730
\end{array}\right] \text {, }
$$

$$
\begin{aligned}
\boldsymbol{A}(i, i+3)_{(1 \longleftrightarrow 4) / 4} & \\
& =\left[\begin{array}{lrrr}
5.6760 & & & \\
& 0.5810 & & \\
& & 4.2730 & \\
& & & 1.3430
\end{array}\right] .
\end{aligned}
$$

Using the SeDuMi package [28] to solve the given set of LMIs (110)-(116) the LMI variables are

$$
\begin{aligned}
\boldsymbol{P} & =\operatorname{diag}\left[\begin{array}{llll}
0.7015 & 0.0570 & 0.1416 & 0.0521
\end{array}\right], \\
\boldsymbol{Q} & =\operatorname{diag}\left[\begin{array}{llll}
0.1937 & 0.0262 & 0.0795 & 0.0094
\end{array}\right], \\
\boldsymbol{R}_{1} & =\operatorname{diag}\left[\begin{array}{llll}
1.5314 & 0.0853 & 2.9122 & 0.0469
\end{array}\right], \\
\boldsymbol{R}_{2} & =\operatorname{diag}\left[\begin{array}{llll}
0.5444 & 0.3043 & 5.2296 & 1.3593
\end{array}\right] .
\end{aligned}
$$

The control law gain matrix is computed by using (117) as

$$
\begin{aligned}
\boldsymbol{k}_{1}^{T} & =\left[\begin{array}{llll}
2.1831 & 1.4967 & 20.5689 & 0.9003
\end{array}\right], \\
\boldsymbol{k}_{2}^{T} & =\left[\begin{array}{llll}
0.7761 & 5.3383 & 36.9371 & 26.0694
\end{array}\right], \\
\boldsymbol{K} & =\left[\begin{array}{llll}
2.1831 & 1.4967 & 20.5689 & 0.9003 \\
0.7761 & 5.3383 & 36.9371 & 26.0694
\end{array}\right],
\end{aligned}
$$

which imply the stable strictly Metzler matrix of closed-loop dynamics (96)

$$
\boldsymbol{A}_{c}=\left[\begin{array}{cccc}
-3.4820 & 0.0473 & 5.1949 & 5.1478 \\
0.4413 & -4.4834 & 0.1321 & 0.0947 \\
1.0178 & 4.0881 & -8.0497 & 5.0626 \\
0.0100 & 2.1652 & 0.4810 & -2.5577
\end{array}\right] \text {, }
$$




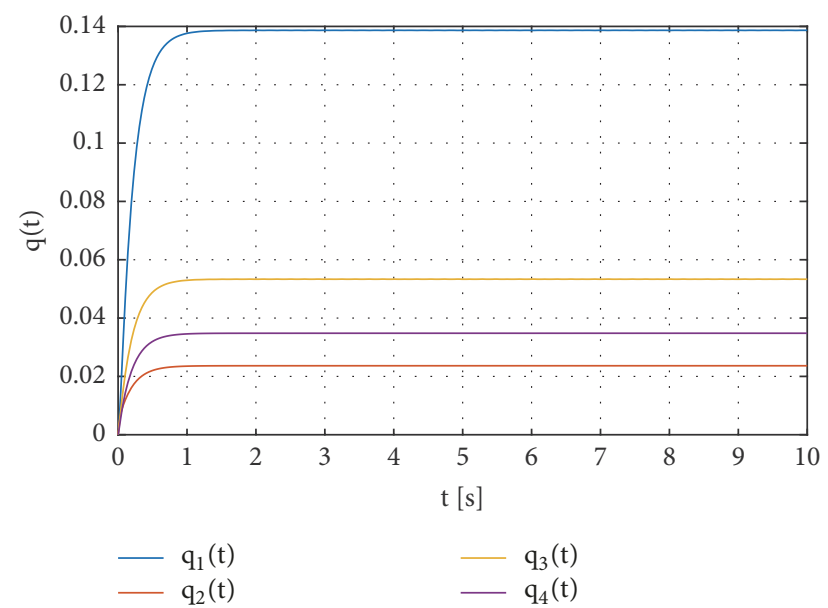

FIgURE 1: The system state variable responses.

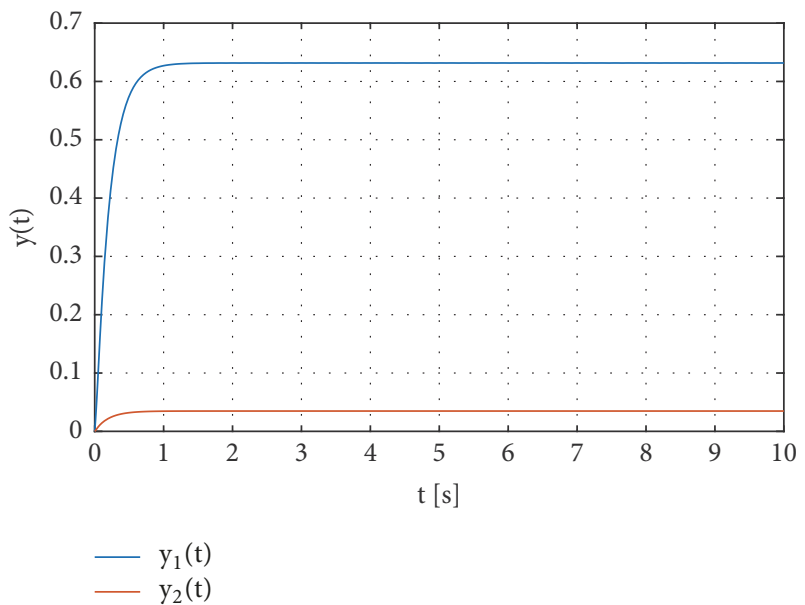

FIGURE 2: The system output variable responses.

with the eigenvalue spectrum

$$
\rho\left(\boldsymbol{A}_{c}\right)=\{-0.5008,-9.1379,-4.4670 \pm 1.2281 \mathrm{i}\} .
$$

Analyzing the numerical results, it is evident that in this case the stabilization of the unstable strictly Metzlerian system principally changes the value of the unstable mode, while stable modes are modified very little. It is also obvious that the strictly Metzler matrix $\boldsymbol{A}_{c}$ is stable but not diagonally dominant.

The obtained results are illustrated in Figures 1 and 2, where the state variables vector $\boldsymbol{q}(t)$ as well as the output variables vector $\boldsymbol{y}(t)$ are positive when the input of the closedloop system is $\boldsymbol{u}(t)=-\boldsymbol{K} \boldsymbol{q}(t)+\boldsymbol{w}(t)$ with the positive forced vector $\boldsymbol{w}^{T}(t)=\left[\begin{array}{ll}0.5 & 0.3\end{array}\right]$.

One can verify that these results are perfectly comparable with the ones obtained by the methodology constructed for the discrete-time positive LTI systems for the associated continuous-time and discrete-time system models [29].
To design the strictly Metzlerian observer, the auxiliary parameters are constructed as

$$
\begin{aligned}
& \boldsymbol{A}(i+1, i)_{(1 \longleftrightarrow 4) / 4} \\
& \quad=\left[\begin{array}{llll}
0.5810 & & & \\
& 4.2730 & & \\
& & 1.3430 & \\
& & & 5.6760
\end{array}\right],
\end{aligned}
$$

$\boldsymbol{A}(i+2, i)_{(1 \longleftrightarrow 4) / 4}$

$$
=\left[\begin{array}{llll}
1.0670 & & & \\
& 2.2730 & & \\
& & 6.7150 & \\
& & & 0.6750
\end{array}\right] \text {, }
$$

$$
\begin{aligned}
& \boldsymbol{A}(i+3, i)_{(1 \longleftrightarrow 4) / 4} \\
& \quad=\left[\begin{array}{llll}
0.0480 & & & \\
& 0.2080 & & \\
& & 2.0500 & \\
& & & 5.8930
\end{array}\right] .
\end{aligned}
$$

Therefore, the set of LMIs (133)-(139) is satisfied with the LMI variables

$$
\begin{aligned}
\boldsymbol{V} & =\operatorname{diag}\left[\begin{array}{llll}
0.1869 & 0.5680 & 0.3436 & 0.3567
\end{array}\right], \\
\boldsymbol{U} & =\operatorname{diag}\left[\begin{array}{llll}
0.2032 & 0.5450 & 0.3678 & 0.5954
\end{array}\right], \\
\boldsymbol{W}_{1} & =\operatorname{diag}\left[\begin{array}{llll}
0.0294 & 0.0583 & 0.0676 & 0.0016
\end{array}\right], \\
\boldsymbol{W}_{2} & =\operatorname{diag}\left[\begin{array}{llll}
0.8587 & 0.2377 & 1.6965 & 0.9121
\end{array}\right] .
\end{aligned}
$$

The observer gain matrix is computed by using (140) as

$$
\begin{aligned}
\boldsymbol{j}_{1} & =\left[\begin{array}{l}
0.1573 \\
0.1026 \\
0.1967 \\
0.0046
\end{array}\right], \\
\boldsymbol{j}_{2} & =\left[\begin{array}{l}
4.5958 \\
0.4186 \\
4.9373 \\
2.5572
\end{array}\right], \\
\boldsymbol{J} & =\left[\begin{array}{ll}
0.1573 & 4.5958 \\
0.1026 & 0.4186 \\
0.1967 & 4.9373 \\
0.1967 & 2.5572
\end{array}\right],
\end{aligned}
$$




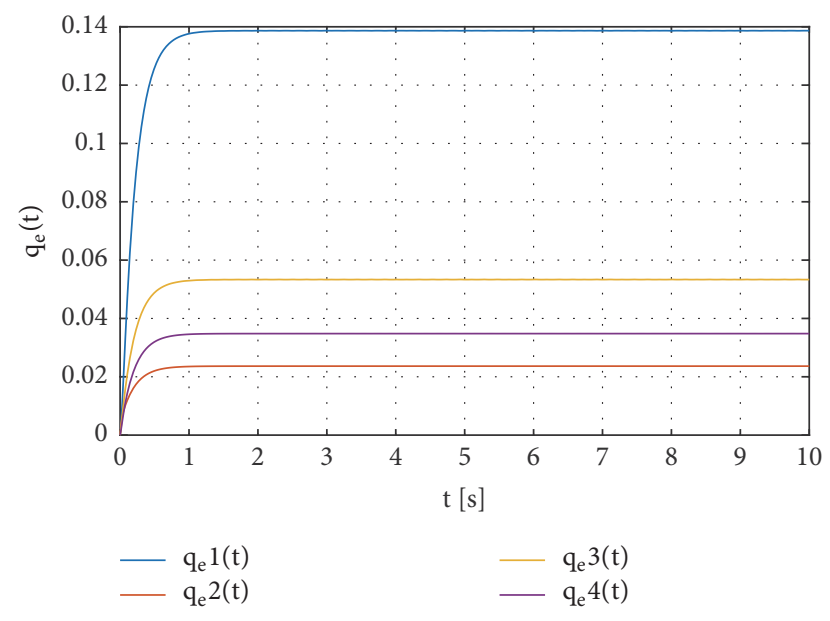

FIGURE 3: The system state variable estimates.

which leads to the stable strictly Metzler matrix of the observer dynamics (100)

$$
\boldsymbol{A}_{e}=\left[\begin{array}{cccc}
-4.0092 & 0.0507 & 6.5577 & 1.0802 \\
0.1706 & -4.3926 & 1.9474 & 0.2564 \\
0.2802 & 4.0763 & -6.8507 & 0.9557 \\
0.0026 & 2.2684 & 1.3384 & -4.6612
\end{array}\right],
$$

with the eigenvalue spectrum

$$
\rho\left(A_{e}\right)=\{-1.3666,-4.6855,-4.9957-8.8659\} .
$$

Analyzing the results, it is evident that the stable strictly Metzlerian observer is designed to the unstable strictly Metzlerian system that is the observer system matrix $\boldsymbol{A}_{e}$ is stable strictly Metzler, but not diagonally dominant.

In Figure 3, the response of the strictly Metzlerian observer in estimating the closed-loop system state variable is presented, acting under the same simulation conditions as the above presented for the closed-loop system. Since the state variable estimation errors can be practically neglected, no other responses of this stable Metzlerian observer are presented.

In addition, the example illustrates that at the large positive values of some elements outside the diagonal of the strictly Metzler matrix of the system $\boldsymbol{A}$, neither the closedloop system matrix $\boldsymbol{A}_{c}$ nor the observer system matrix $\boldsymbol{A}_{e}$ is diagonally dominant, although they are stable strictly Metzler matrices. However, the results of our other simulations show that if the system matrix $\boldsymbol{A}$ is diagonally dominant strictly Metzler matrix, even the matrices $\boldsymbol{A}_{c}$ and $\boldsymbol{A}_{e}$ are diagonally dominant to the strictly Metzler matrix.

\section{Concluding Remarks}

A novel approach is presented in the paper to address the problem of effectively computing a state-feedback control law gain that makes the strictly Metzlerian system in closedloop to be positive and stable, as well as of recounting the observer gain that establish the stable strictly Metzlerian structures of Luenberger observers. Based on a stable strictly Metzler matrix, algebraic constraints implying from linear programming approach are reformulated as a set of LMIs and replenished by the Lyapunov matrix inequality in the sense of the second Lyapunov method. It is derived that all matrix variables associated with this LMIs ensemble have to be positive definite and diagonal. The proposed approach provides a numerically reliable computational framework, as illustrated using the numerical example, and might be extended to other particular cases.

The example also illustrates that at the large positive values of some elements outside the diagonal of the strictly Metzler matrix of the system $\boldsymbol{A}$, neither the closed-loop system matrix $\boldsymbol{A}_{c}$ nor the observer system matrix $\boldsymbol{A}_{e}$ is diagonally dominant, although they are stable strictly Metzler matrices. The results of our other simulations show that if the system matrix $\boldsymbol{A}$ is diagonally dominant strictly Metzler matrix, the matrices $\boldsymbol{A}_{c}$ and $\boldsymbol{A}_{e}$ are diagonally dominant strictly Metzler matrix. Further research topics include this problem as well as the problems of forced mode in positive systems, discrete-time linear positive structures, and nonlinear positive systems.

\section{Conflicts of Interest}

The authors declare that there are no conflicts of interest regarding the publication of this paper.

\section{Acknowledgments}

The work presented in this paper was supported by VEGA, the Grant Agency of the Ministry of Education, and the Academy of Science of Slovak Republic, under Grant no. $1 / 0608 / 17$. This support is very gratefully acknowledged.

\section{References}

[1] F. Cacace, L. Farina, R. Setola, and A. Germani, Eds., Positive systems, vol. 471 of Lecture Notes in Control and Information Sciences, Springer, Cham, 2016.

[2] J. M. Carnicer, J. M. Pena, and R. A. Zalik, "Strictly totally positive systems," Journal of Approximation Theory, vol. 92, no. 3, pp. 411-441, 1998.

[3] O. Pastravanu and M. H. Matcovschi, "M, $\beta$-stability of positive linear systems," Mathematical Problems in Engineering, vol. 2016, Article ID 9605464, p. 11, 2016.

[4] X. Chen, J. Lam, P. Li, and Z. Shu, " $l_{1}$-induced norm and controller synthesis of positive systems," Automatica, vol. 49, no. 5, pp. 1377-1385, 2013.

[5] N. K. Son and D. Hinrichsen, "Robust stability of positive continuous time systems," Numerical Functional Analysis and Optimization, vol. 17, no. 5-6, pp. 649-659, 1996.

[6] T. Kaczorek, "Stability and stabilization of positive fractional linear systems by state-feedbacks," Bulletin of the Polish Academy of Sciences-Technical Sciences, vol. 58, no. 4, pp. 537554, 2010. 
[7] J. Back and A. Astolfi, "Design of positive linear observers for positive linear systems via coordinate transformations and positive realizations," SIAM Journal on Control and Optimization, vol. 47, no. 1, pp. 345-373, 2008.

[8] Z. Shu, J. Lam, H. Gao, B. Du, and L. Wu, "Positive observers and dynamic output-feedback controllers for interval positive linear systems," IEEE Transactions on Circuits and Systems I: Regular Papers, vol. 55, no. 10, pp. 3209-3222, 2008.

[9] B. Shafai and A. Oghbaee, "Positive quadratic stabilization of uncertain linear system," in Proceedings of the 2014 IEEE MultiConference on Systems and Control, CCA 2014, pp. 1412-1417, Antibes, France, October 2014.

[10] B. Shafai, R. Ghadami, and A. Oghbaee, "Constrained stabilization with maximum stability radius for linear continuoustime systems," in Proceedings of the 52nd IEEE Conference on Decision and Control, CDC 2013, pp. 3415-3420, Florence, Italy, December 2013.

[11] J.-E. Feng, J. Lam, P. Li, and Z. Shu, "Decay rate constrained stabilization of positive systems using static output feedback," International Journal of Robust and Nonlinear Control, vol. 21, no. 1, pp. 44-54, 2011.

[12] J. Shen and J. Lam, "On static output-feedback stabilization for multi-input multi-output positive systems," International Journal of Robust and Nonlinear Control, vol. 25, no. 16, pp. 3154-3162, 2015.

[13] M. Ait Rami and F. Tadeo, "Linear programming approach to impose positiveness in closed-loop and estimated states," in Proceedings of the 16th International Symposium on Mathematical Theory of Networks and Systems, pp. 2470-2477, Kyoto, Japan, 2006.

[14] M. A. Rami and F. Tadeo, "Controller synthesis for positive linear systems with bounded controls," IEEE Transactions on Circuits and Systems II: Express Briefs, vol. 54, no. 2, pp. 151-155, 2007.

[15] H. Gao, J. Lam, C. Wang, and S. Xu, "Control for stability and positivity: Equivalent conditions and computation," IEEE Transactions on Automatic Control, vol. 52, no. 9, pp. 540-544, 2005.

[16] D. Krokavec and A. Filasová, "On control of discrete-time LTI positive systems," Applied Mathematical Sciences, vol. 11, no. 50, pp. 2459-2476, 2017.

[17] L. Farina and S. Rinaldi, Positive Linear Systems: Theory and Applications, John Wiley \& Sons, New York, NY, USA, 2000.

[18] D. G. Luenberger, Introduction to Dynamic Systems. Theory, Models and Applications, John Wiley \& Sons, New York, NY, USA, 1979.

[19] A. Berman, M. Neumann, and R. J. Stern, Nonnegative Matrices in Dynamic Systems, John Wiley \& Sons, New York, NY, USA, 1989.

[20] N. Dautrebande and G. Bastin, "Positive linear observers for positive linear systems," in Proceedings of the European Control Conference ECC'99, Paper ID F371, Karlsruhe, Germany, 1999.

[21] T. Kaczorek, Positive $1 D$ and $2 D$ Systems, Springer-Verlag, London, UK, 2002.

[22] R. A. Horn and C. R. Johnson, Matrix Analysis, Cambridge University Press, New York, NY, USA, 2013.

[23] P. D. Leenheer and D. Aeyels, "Stabilization of positive linear systems," Systems \& Control Letters, vol. 44, no. 4, pp. 259-271, 2001.

[24] C. A. Crusius and A. Trofino, "Sufficient LMI conditions for output feedback control problems," IEEE Transactions on Automatic Control, vol. 44, no. 5, pp. 1053-1057, 1999.
[25] G. Birkhoff and S. Mac Lane, A survey of Modern Algebra, Macmillan Publishing, New York, USA, 1977.

[26] D. J. S. Robinson, An Introduction to Abstract Algebra, De Gruyter Textbook, Walter de Gruyter, Berlin, Germany, 2003.

[27] W. M. Haddad and V. Chellaboina, Nonlinear Dynamical Systems and Control. A Lyapunov-Based Approach, Princeton University Press, Princeton, NJ, USA, 2008.

[28] D. Peaucelle, D. Henrion, Y. Labit, and K. Taitz, User's Guide for SeDuMi Interface, LAAS-CNRS, Toulouse, France, 2002.

[29] D. Krokavec and A. Filasová, "Stabilization of discrete-time LTI positive systems," Archives of Control Sciences, vol. 27, no. 4, pp. 575-594, 2017. 


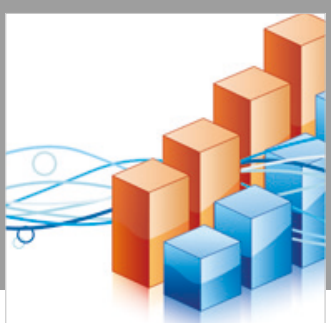

Advances in

Operations Research

\section{-n-m}
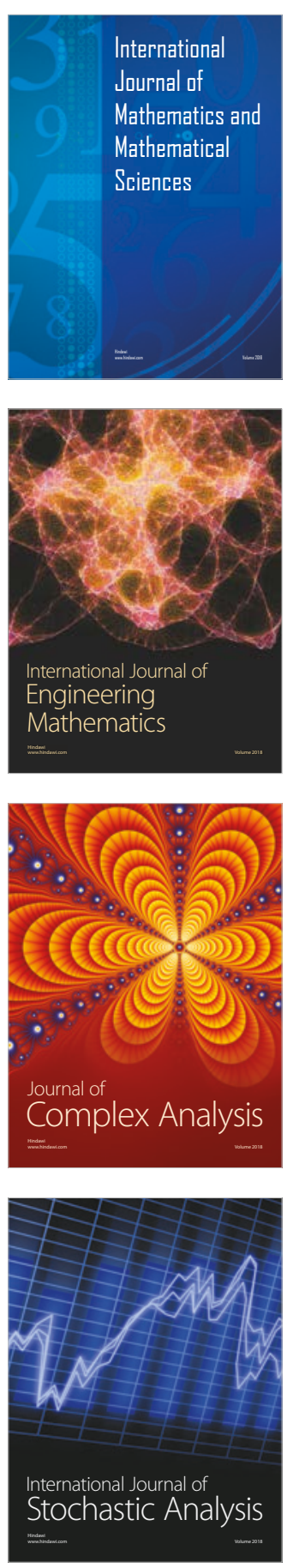
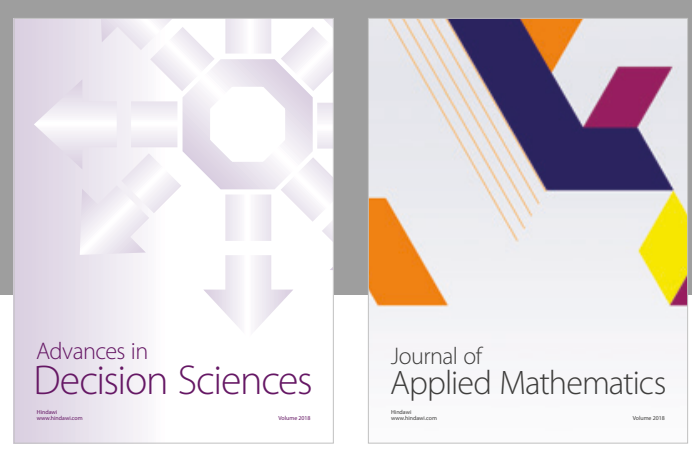

Journal of

Applied Mathematics
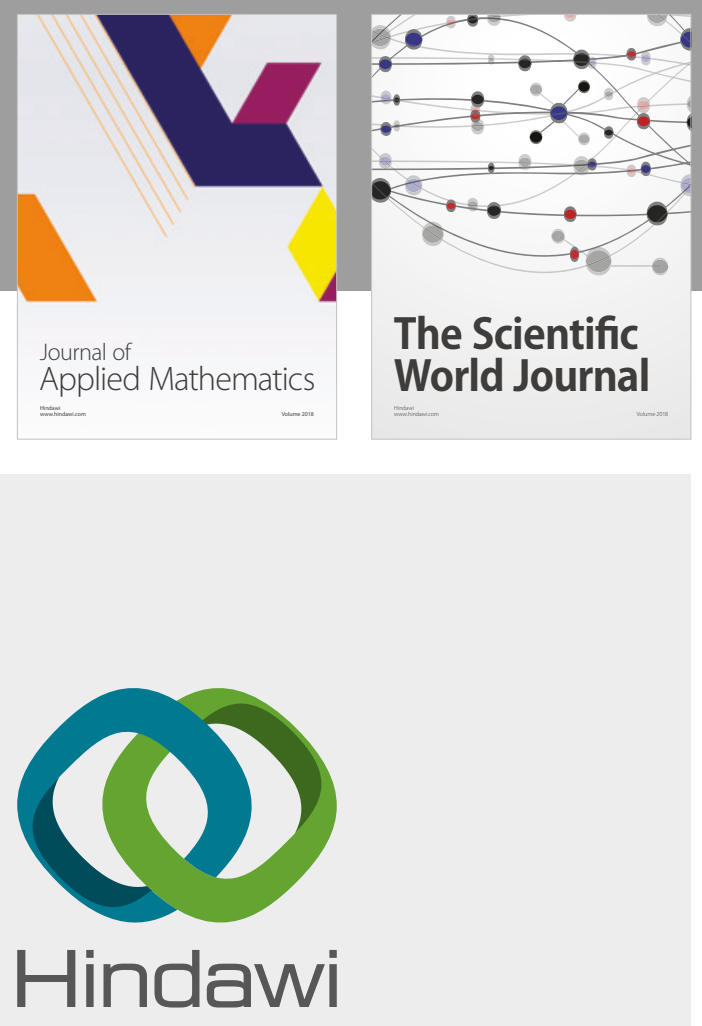

Submit your manuscripts at

www.hindawi.com

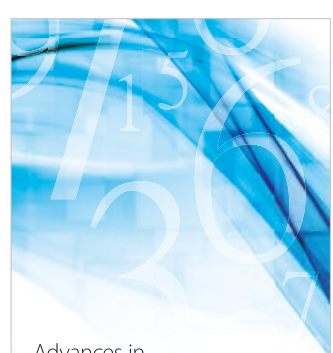

Advances in
Numerical Analysis
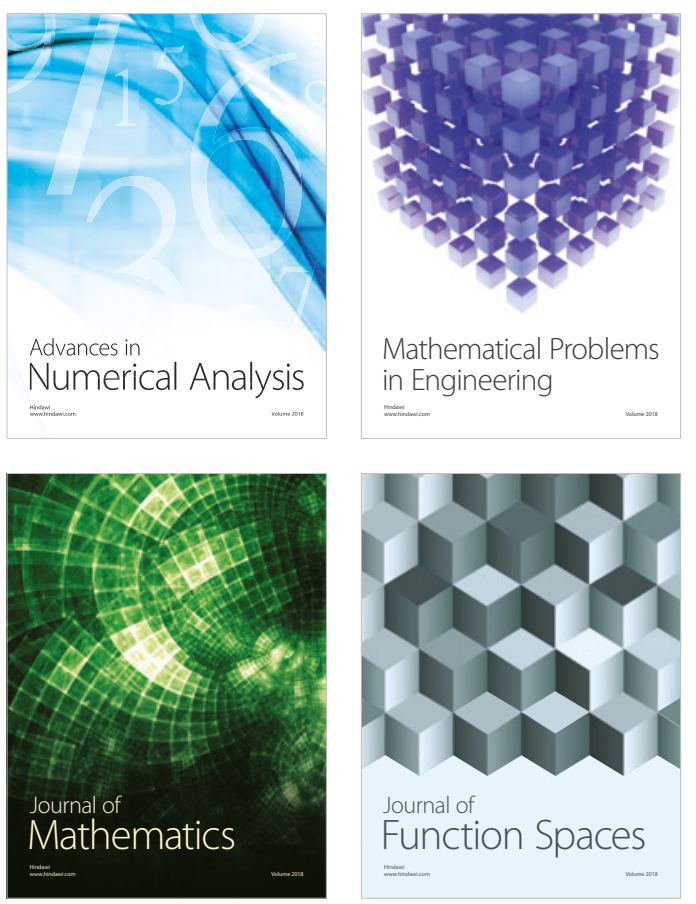

Mathematical Problems in Engineering

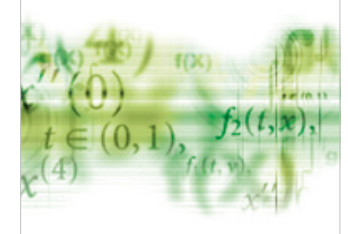

International Journal of

Differential Equations

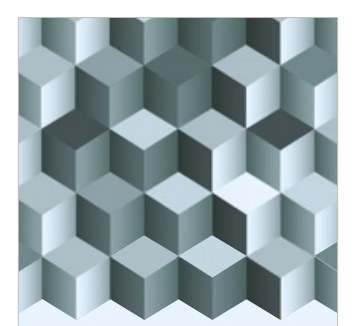

Journal of

Function Spaces

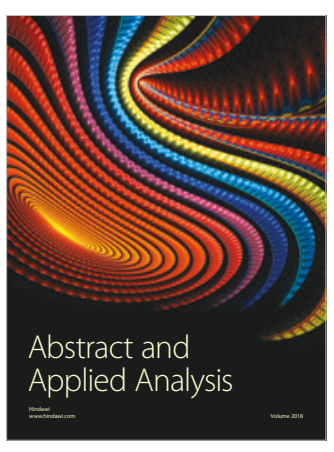

The Scientific

World Journal

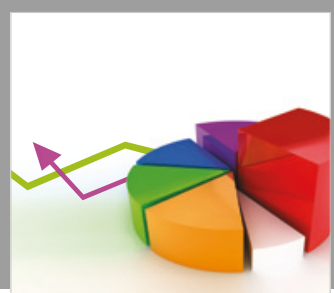

Journal of

Probability and Statistics
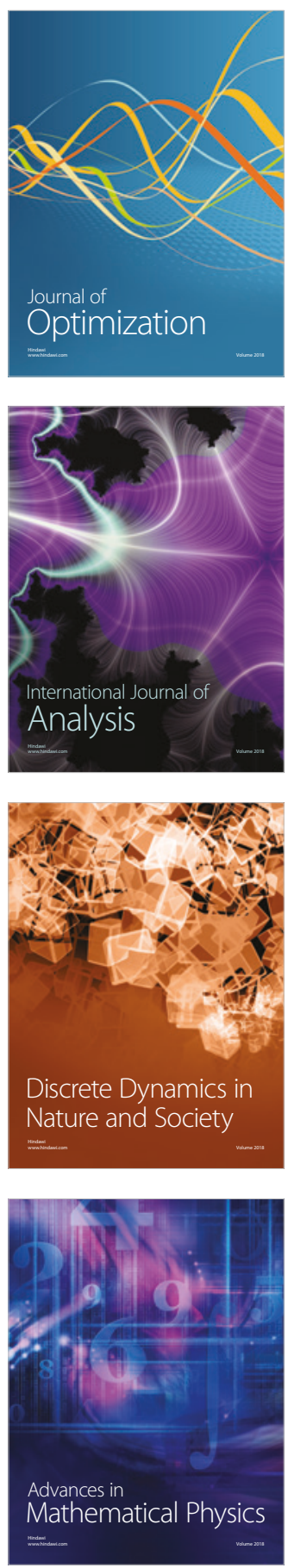